\title{
Harmonic-cavity stabilization of longitudinal coupled-bunch instabilities with a nonuniform fill
}

\author{
F. J. Cullinan, Å. Andersson, and P. F. Tavares \\ MAX IV Laboratory, Lund University, SE-2100 Lund, Sweden
}

(Received 9 April 2020; accepted 7 July 2020; published 14 July 2020)

\begin{abstract}
In synchrotrons, nonuniform fill patterns, which give rise to beam phase transients and a spread in synchrotron tune between bunches, have been observed to damp longitudinal coupled-bunch instabilities driven by higher-order modes in rf cavities. The transients are especially large in the presence of Landau cavities, which are used commonly in storage-ring light sources and particularly in the new generation of diffraction-limited storage rings. A method has recently been devised to predict the beam transient including complex form factors for the different bunches. This has now been extended to accurately predict the growth-rates and oscillation frequencies of coupled-bunch modes for arbitrary fill patterns, taking the individual complex form factors and equilibrium phases of the different bunches into account. In this paper, the extended method is presented and the theory is outlined. For a case with significant transient beam loading, predictions of the resulting beam transient and bunch profiles are compared to measurements. Predictions of coupled-bunch mode behavior are then benchmarked against results from the macroparticle tracking code MBTRACK with good agreement. Finally, the method is used to predict the behavior of coupled-bunch modes as a function of the fields in passive Landau cavities.
\end{abstract}

DOI: 10.1103/PhysRevAccelBeams.23.074402

\section{INTRODUCTION}

In synchrotron storage rings, rf cavities are used to return the energy that a charged-particle beam has lost to synchrotron radiation. They also contain the charged particles in bunches where they execute stable synchrotron oscillations inside an enclosing potential. In electron storage rings used as dedicated sources of synchrotron radiation, Landau cavities are sometimes used to flatten the center of this potential [1], thereby lengthening the electron bunches. Increasing the bunch volume in such a way increases the Touschek beam lifetime and reduces emittance blow-up due to intrabeam scattering [2]. This is particularly important in the latest generation of synchrotron light sources, so-called diffraction-limited storage rings (DLSRs), which employ multibend achromats to reach ultralow horizontal emittances.

Commonly, the fundamental mode of a Landau cavity is loaded by the beam itself and has a high quality factor so that its fields have a significant effect on the rf potential. In this way, the fundamental mode can be seen as a long-range wakefield that has a significant static effect. Throughout

\footnotetext{
*francis.cullinan@maxiv.lu.se

Published by the American Physical Society under the terms of the Creative Commons Attribution 4.0 International license. Further distribution of this work must maintain attribution to the author(s) and the published article's title, journal citation, and DOI.
}

this paper, beam loading of long-range wakefields is described as either "static" or "dynamic." Static loading refers to cases where the centroid and form of the chargedparticle bunches are stationary relative to their rf bucket and the wakefield is excited at an exact harmonic of the machine revolution frequency. Dynamic loading is where there is oscillation of the bunches within their rf buckets and so the wakefield is excited at a revolution harmonic plus or minus the oscillation frequency depending on the sign of the phase advance from one bunch to the next.

The fundamental mode of a Landau cavity also couples the motion of the different bunches and in bunchlengthening mode, is tuned in such a way that it destabilizes the Robinson mode (motion of all bunches synchronized). Machines are therefore designed so that this is compensated by the Robinson damping from the beam-loaded fundamental mode in the main rf cavities, which are typically tuned to match the beam-loaded cavity to the rf power generator. The dynamic effects of the Landau-cavity fundamental mode are in this way minimized.

In both the main and Landau cavities, the beam also excites unwanted higher-order modes (HOMs) whose resonant frequencies could be close to revolution harmonics. Without selective damping, these HOMs can have large quality factors comparable to that of the fundamental cavity mode. If every rf bucket in the machine is filled with a bunch of exactly the same charge (an even/uniform fill), the effect of the static loading of these modes is negligible because the beam has no frequency content at the revolution harmonics that are not also harmonics of the rf and if a 
HOM has a resonant frequency that is close to an $\mathrm{rf}$ harmonic, its shunt impedance is typically small compared to that of the cavity fundamental modes. However, a HOM can excite a coupled-bunch mode that is nearby in frequency (and damp its complement $h-m$ where $h$ is the machine harmonic number and $m$ is the coupled-bunch mode number). If the resulting growth rate is larger than the damping rate (which may come from synchrotron-radiation damping or from a feedback system), that coupled-bunch mode is unstable. The synchrotron oscillations will typically saturate at some amplitude due to the nonlinearities in the longitudinal dynamics but this usually constitutes a significant increase in the energy spread in the beam with a resulting deterioration of the synchrotron-radiation brightness. The dynamic loading of a HOM is therefore more relevant to stability than the static loading.

In addition to mitigating the negative effects of Touschek and intrabeam scattering, bunch lengthening provided by Landau cavities also reduces the growth rates of HOMdriven coupled-bunch modes. This is because longer bunches have a narrower frequency spectrum with lower amplitude at the typically high frequencies of the HOMs. Landau cavities also introduce Landau damping, another way of preventing the destabilization of coupled-bunch modes. Landau cavities make the rf potential less harmonic and so the frequency of synchrotron oscillations becomes dependent on the amplitude and in this way, the spread of synchrotron frequencies among the particles in a bunch is increased. A spread in synchrotron frequency between the bunches can also be introduced by varying the amount of charge in each rf bucket. The resulting transient beamloading of the fundamental modes in the Landau and main cavities means that their fields are modulated on the time scale of a single revolution. Consequently, each bunch is enclosed in a slightly different potential and finds a different equilibrium rf phase where it gains no net energy gain per turn. This has a significant effect on the dynamics of coupled-bunch modes, as has been observed experimentally at ELETTRA [3] and ESRF [4] where, in both cases, the effect was positive. Static loading of the HOMs is also introduced because the machine fill is no longer uniform and so the frequency spectrum of the beam includes certain revolution harmonics, depending on the exact fill pattern.

This paper explores the effects of the transient beam loading of Landau cavities in a synchrotron where HOMdriven coupled-bunch instabilities dominate in the longitudinal plane. Both the static and dynamic effects are explored. In order to explore the former, a time-domain matrix-based method, first presented in [5], is employed. It is then extended to investigate dynamic effects. Similar analyses of the latter can be found in [4,6-8] but in all those references, the assumption is made that, while not being of equal charge (some bunches may have zero charge), the different bunches are equally spaced in time (by one rf period). This assumption is necessary for the conversion to
TABLE I. Nominal parameters of the MAX IV $3 \mathrm{GeV}$ ring in delivery including the definition used for the shunt impedance $R_{s}$ where $V$ and $P$ are the voltage and power in the cavity respectively.

\begin{tabular}{lc}
\hline \hline Parameter & Value \\
\hline Energy $E_{0}$ & $3 \mathrm{GeV}$ \\
Circumference & $528 \mathrm{~m}$ \\
rf frequency $f_{\text {rf }}$ & $99.931 \mathrm{MHz}$ \\
rf voltage $V_{0}$ & $1.251 \mathrm{MV}$ \\
Current & $250 \mathrm{~mA}$ \\
Harmonic number $h$ & 176 \\
Energy loss per turn $U_{0}$ & $363.8 \mathrm{keV}$ \\
Radiation damping time $\mathcal{T}_{0}$ & $25.194 \mathrm{~ms}$ \\
Momentum compaction $\alpha_{c}$ & 0.000306 \\
Natural normalized energy spread & 0.000769 \\
Landau cavity (LC) harmonic & 3 \\
Total LC shunt impedance $R_{s}=V^{2} /(2 P)$ & $8.25 \mathrm{M} \Omega$ \\
LC quality factor & 20800 \\
\hline \hline
\end{tabular}

the frequency domain, where the problem is considered in terms of coupled-bunch modes ordered by resonant frequency. Therefore, if the whole analysis is restricted to the time domain, this limitation can be avoided. Once the static beam-loading transient has been evaluated, including equilibrium longitudinal profiles and form factors for each bunch, the results can be used to calculate the stability of the HOM-driven coupled-bunch modes. This is done using an extension to the method that includes the uneven spacing and spread in synchrotron tune between bunches and, unlike previous works, the individual bunch form factors as well. The output of the first step can be used directly as the input to the second step so no post-processing is required. In this way, HOM-driven coupled-bunch modes in a synchrotron with any arbitrary fill pattern can be fully evaluated in a computationally efficient way. The form factors used in both steps can either be complex (have both real and imaginary components) or purely real of which, the former is more accurate. The latter is referred to as the scalar approximation in [9] and is also discussed in [5]. In this paper, where form factors are used, they are always complex.

The $3 \mathrm{GeV}$ ring at the MAX IV laboratory in Lund, Sweden, the first of the aforementioned DLSRs, has been used to test this method. The relevant machine parameters are listed in Table I. The MAX IV $3 \mathrm{GeV}$ ring is also unique in that Landau cavities were included from the conceptual design stage for the reasons mentioned previously and with the knowledge that it would help stabilize the beam against coupled-bunch instabilities both longitudinally and transversely [10] by introducing synchrotron-tune spread and reducing the overlap of the beam spectrum with that of the main driving impedances by lengthening the bunches. It is also a machine where HOMs in the normal-conducting 
main and Landau cavities (which have no HOM dampers) are the main driving forces for longitudinal coupled-bunch mode instabilities. Extensive temperature-tuning has been necessary to move the HOMs away from the revolution harmonics and obtain stable beam with the help of Landau damping and synchrotron-radiation damping. No feedback is used to stabilize the beam longitudinally except for a mode-0 damper $[11,12]$.

In Sec. II, the theory behind the new two-step method is outlined, starting with a summary of the theory from [5] in Sec. II A that is used for the first step of predicting the static beam transient. This theory has now been extended, as outlined in Sec. II B, to evaluate the growth rates of coupled-bunch modes, the second step. In Sec. III, predictions of the static beam transient are compared with experimental observations. Next, the method for predicting the growth rates of HOM-driven coupled-bunch modes is benchmarked against the macroparticle-tracking code MBTRACK [13] in Sec. IV. Finally, in Sec. V, the effect of the Landau cavities at the MAX IV $3 \mathrm{GeV}$ ring is investigated by evaluating the growth rates of coupledbunch modes for different Landau-cavity fields. Landau damping due to the synchrotron-frequency spread within each bunch (intrabunch) is not treated by the theory and is neglected. As shown later, for the particular nonuniform fill and parameter range studied here, the effect is small compared to the damping due to bunchto-bunch (interbunch) synchrotron-frequency spread and this is likely to be the case for most nonuniform fills. Theories developed by Krinsky [14], later followed up by Lindberg [15] and Venturini [16], cover, for even fill patterns, cases where the intrabunch frequency spread dominates and in particular, the flat-potential condition defined in $[1,9]$.

Another important consideration that is not treated by the theory is the short-range wakefield and the single-bunch instabilities that may arise as a result. It is important to evaluate these independently because, in some cases, they may be a limiting factor in the use of Landau cavities for bunch lengthening [17].

\section{THEORY}

\section{A. Static transient}

The theoretical method used to determine the static transient in a nonuniform machine fill is outlined in [5] and is briefly summarized here. Based on an iterative matrix formulation, it is a self-consistent, semianalytical method for determining the time offsets of the different bunches relative to some initial estimate of the synchronous phase $\phi_{s}$ of the main rf. The longitudinal charge profiles of the different bunches are also determined. The extension for determining the growth rates of HOM-driven coupledbunch modes is outlined in Sec. II B and makes use of many of the same definitions.
The aforementioned time offsets are collected in a vector $\tau_{l}$ and starting from $\tau_{l}=0$, a correction $\delta \tau_{l}$ can be estimated, which is the time offset from some point of equilibrium where the net energy change per turn of a single particle is zero. The sources of energy change that are dependent on $\tau_{l}$, namely the voltage due to the longrange wakefield from another bunch $V_{b}\left(\tau_{l}, \tau_{j}\right)$ and the rf voltage $V_{\mathrm{rf}}\left(\tau_{l}\right)=V_{0} \sin \left(2 \pi f_{\mathrm{rf}} \tau_{l}+\phi_{s}\right)$, are Taylor expanded to first order and the first-order terms are collected on the left-hand side of the following matrix equation:

$$
\begin{aligned}
& \sum_{j=0}^{h-1} \operatorname{Re}\left[\frac{\partial V_{b}}{\partial \tau_{j}}\left(\tau_{l}, \tau_{j}\right)+V_{\mathrm{rf}}^{\prime}\left(\tau_{j}\right) \delta_{j l}\right] \delta \tau_{j} \\
& =-\frac{U_{0}}{e_{0}}+V_{\mathrm{rf}}\left(\tau_{l}\right)-\sum_{j=0}^{h-1} \operatorname{Re}\left[V_{b}\left(\tau_{l}, \tau_{j}\right)\right]-k\left|F_{l}\right| q_{l} .
\end{aligned}
$$

The right-hand side contains the constant terms from the Taylor expansions and the energy lost to synchrotron radiation $U_{0}$ and to the short-range wake according to the fundamental theorem of beam loading $k\left|F_{l}\right| q_{l}$. Here, $e_{0}$ is the elementary charge, $\delta_{l j}$ is the Kronecker delta, $k$ is the loss factor defined below, $q_{l}$ is the charge of bunch $l$ (empty buckets may be included by setting $q_{l}=0$ ) and $F_{l}$ is its form factor.

The beam-loading voltage $V_{b}\left(\tau_{l}, \tau_{j}\right)$ in bucket $l$ due to the charge in bucket $j$ is calculated by summing the longrange wake potential from that bucket over infinite turns. Here, only resonant wakefields are considered and these can each be parametrized by a resonant angular frequency $\omega_{r}$, a damping parameter $\alpha$ and a point-charge loss factor $k$. A complex wake function is used because this simplifies the subsequent mathematical expressions but the imaginary part has no physical significance and is ultimately neglected in Eq. (1). The total wake function is then given by

$$
W(t)=-\sum_{m=0}^{M} 2 k_{m} e^{-\left(i \omega_{r, m}+\alpha_{m}\right) t}
$$

where the summation is over $M$ different resonances whose individual parameters are indexed with the $m$ subscript. From here on, only one resonance is considered so that the summation over $m$ can be dropped but it can easily be reintroduced later at any stage. The parameters $k$ and $\alpha$ are used for compactness and can be calculated from the resonant frequency, the loaded shunt impedance $R_{L}$ and the quality factor $Q_{L}$ of a resonator as

$$
\begin{gathered}
k=\frac{\omega_{r} R_{L}}{2 Q_{L}} \\
\alpha=\frac{\omega_{r}}{2 Q_{L}} .
\end{gathered}
$$


The form factor of each bunch must also be calculated at the resonant frequency of the wake as

$$
F_{l}=\left|F_{l}\right| e^{i \varphi_{l}}=\int_{-\infty}^{\infty} e^{-i \omega_{r}\left(\tau-\tau_{l}\right)} \rho\left(\tau-\tau_{l}\right) d \tau
$$

where $\rho$ is the normalized charge density of the bunch over the time offset $\tau$. The form factor approximates the convolution of the wake function with the longitudinal bunch profile $\rho$ for the case of a resonant wake function whose amplitude does not change significantly during the length of the bunch. The form-factor phase $\phi_{l}$ accounts for the phase shift that results from convolution of the wake function with an asymmetric bunch profile. An in-depth discussion of the form factor can be found in [9].

Similarly to in [18], the infinite sum to determine the voltage in bucket $l$ due to the charge in bucket $j$ is evaluated to give

$$
\begin{aligned}
V_{b}\left(\tau_{l}, \tau_{j}\right) & =\sum_{n=0}^{\infty} F_{l} q_{l} W\left[n T_{0}+\Delta t\left(\tau_{l}, \tau_{j}\right)\right] \\
& =\frac{-2 k F_{l} q_{l}}{1-e^{\left(-i \omega_{r}-\alpha\right) T_{0}}} e^{-\left(i \omega_{r}+\alpha\right) \Delta t\left(\tau_{l}, \tau_{j}\right)}
\end{aligned}
$$

where the time between the two bunches $\Delta t\left(\tau_{l}, \tau_{j}\right)$ is given by

$\Delta t\left(\tau_{l}, \tau_{j}\right)=\left\{\begin{array}{ll}\tau_{j}-\tau_{l}+(l-j+h) / f_{\mathrm{rf}} & \text { for } l \leq j \\ \tau_{j}-\tau_{l}+(l-j) / f_{\mathrm{rf}} & \text { for } l>j\end{array}\right.$.

The derivative with respect to $\tau_{j}$ is then simply

$$
\frac{\partial V_{b}}{\partial \tau_{j}}\left(\tau_{l}, \tau_{j}\right)=-\left(i \omega_{r}+\alpha\right) V_{b}\left(\tau_{l}, \tau_{j}\right) .
$$

Equation (1) can be iterated, each time recalculating the voltages with the updated equilibrium phases. The form factors can also be calculated and updated every iteration using the method described in [5] and [9]. In this way, a self-consistent solution for the equilibrium time offsets can be obtained.

The charge in a bunch will be distributed around the equilibrium time offset but it may not be the location of the centroid or a peak in the charge distribution in cases where the bunch is overstretched or asymmetric. Furthermore, two equilibrium time offsets may exist within one rf bucket, of which this method will only find one. Provided a complex form factor is used, it does not make the method invalid but it will be more difficult for it to reach convergence in the vector $\tau_{l}$ so another method, such as macroparticle tracking, may be considered.

Reintroducing the summation over multiple resonant wakefields from Eq. (2) allows the consideration of any combination of resonant wakefields from just the fundamental mode of a Landau cavity to the fundamental modes of several Landau cavities and multiple higher order modes in Landau cavities and main cavities. The fundamental mode in the main cavities may also be considered although in this case, the rf voltage $V_{0}$ and phase $\phi_{s}$ would usually have to be adjusted to mimic the effect of a slow rf feedback that keeps the total field amplitude and phase of the main-cavity fundamental mode constant.

\section{B. Coupled-bunch motion}

This section describes the method used to evaluate the dynamic effect of a single long-range resonant wakefield but, as above, can be extended to treat several of them. Attention is turned from evaluating the constant time offset $\tau_{l}$ of each bunch $l$ to its motion $r_{l}$. For this, each bunch is assumed to be a harmonic oscillator whose motion in longitudinal phase space over time is given by

$$
r_{l}(t)=\hat{r}_{l} e^{i \Omega t}
$$

where $\hat{r}$ and $\Omega$ are complex. When the forces acting on bunch $l$ are substituted into the equation of motion for a driven harmonic oscillator [19], the following is obtained:

$$
\begin{aligned}
r_{l}^{\prime \prime} & -i \frac{2}{\mathcal{T}_{0}} r_{l}^{\prime}+\omega_{s, l}^{2} r_{l} \\
= & \frac{\alpha_{c}}{E_{0} T_{0}} \sum_{j=0}^{h-1} \sum_{n=0}^{\infty} r_{j}\left(n T_{0}\right) \operatorname{Re}\left\{F_{l}^{*} F_{j} q_{j} W^{\prime}\left[n T_{0}+\Delta t\left(\tau_{l}, \tau_{j}\right)\right]\right\}
\end{aligned}
$$

Here, $\omega_{s, l}$ is the incoherent synchrotron frequency of the bunch in bucket $l$, which characterizes the if potential for that bucket and, as commonly defined, is the frequency at which individual particles with a small energy offset from the synchronous particle, perform synchrotron oscillations. The right-hand side of Eq. (10) is a sum of the dynamic effect of the long-range wakefield from the charge in the other buckets. As in Section II A, a Taylor expansion has been performed, of which, this is the first order term. The constant term has been dropped because it only gives rise to a constant time offset, which it was the goal of the previous section to evaluate. In this section, it is assumed that the equilibrium time offsets have been evaluated already. The imaginary part of the wake function is again neglected because it has no physical significance. The second term on the left hand side accounts for the radiation damping where $\mathcal{T}_{0}$ is the radiation damping time. Each bunch is still assumed rigid with a fixed form factor so diffusion due to the random nature of radiation emission and quantum excitation is neglected.

The infinite sum over previous turns is evaluated to give analytical expressions for the elements of a matrix that can be inserted into the right-hand side of Eq. (10). 


$$
\begin{aligned}
\mathbf{M}_{l j}= & \sum_{n=0}^{\infty} \operatorname{Re}\left\{F_{l}^{*} F_{j} q_{j} W^{\prime}\left[n T_{0}+\Delta t\left(\tau_{l}, \tau_{j}\right)\right]\right\} \frac{r_{j}\left(n T_{0}\right)}{\hat{r}_{j}} \\
= & -k\left|F_{l}\right|\left|F_{j}\right| q_{j}\left[\frac{\left(i \omega_{r}-\alpha\right) e^{\left(i \omega_{r}-\alpha\right) \Delta t\left(\tau_{l}, \tau_{j}\right)+i\left(\varphi_{j}-\varphi_{l}\right)}}{1-e^{\left(i \omega_{r}-\alpha+i \Omega\right) T_{0}}}\right. \\
& \left.+\frac{\left(-i \omega_{r}-\alpha\right) e^{\left(-i \omega_{r}-\alpha\right) \Delta t\left(\tau_{l}, \tau_{j}\right)-i\left(\varphi_{j}-\varphi_{l}\right)}}{1-e^{\left(-i \omega_{r}-\alpha+i \Omega\right) T_{0}}}\right]
\end{aligned}
$$

In practice, the approximation $\Omega=\left\langle\omega_{s, l}\right\rangle$ is substituted into Eq. (11). This prevents the final eigenvalue equation from having to be solved self-consistently and, as discussed in [15], is justified for the specific case of a HOM impedance. Equation (11) is similar to that found by Thompson and Ruth [6] but with individual complex bunch form-factors included and the general expressions for the time between bunches given by Eq. (7).

Putting Eq. (11) into Eq. (10) and evaluating the lefthand side gives

$$
\left(\Omega^{2}-i \frac{2 \Omega}{\mathcal{T}_{0}}-\omega_{s, l}^{2}\right) \hat{r}_{l}=-\frac{\alpha_{c}}{E_{0} T_{0}} \sum_{j=0}^{h-1} \mathbf{M}_{l j} \hat{r}_{j} .
$$

A common next step would be to invoke the approximation $\Omega \approx\left\langle\omega_{s, l}\right\rangle$ and then write $\Omega^{2}-i \frac{2 \Omega}{T_{0}}-\omega_{s, l}^{2} \approx 2 \omega_{s, l}(\Omega-$ $\left.i / \mathcal{T}_{0}-\omega_{s, l}\right)$ so that the following eigenvalue equation is obtained:

$\Omega \hat{r}_{l}=-\frac{\alpha_{c}}{E_{0} T_{0}} \sum_{j=0}^{h-1} \frac{1}{2 \omega_{s, l}}\left[\mathbf{M}_{l j}+\left(\omega_{s, l}+\frac{i}{\mathcal{T}_{0}}\right) \delta_{j l}\right] \hat{r}_{j}$.

The advantage of this method is that the eigenvalue $\Omega$ is directly the complex coherent frequency of a corresponding mode (eigenvector). However, one of the consequences of bunch-lengthening Landau cavities is that the synchrotron frequency is reduced and may become comparable to the frequency shift due to the other long-range wakefields making the above approximation invalid. It is preferable, therefore, to not make this approximation so that the eigenvalue equation is instead

$$
\left(\Omega^{2}-i \frac{2 \Omega}{\mathcal{T}_{0}}\right) \hat{r}_{l}=-\frac{\alpha_{c}}{E_{0} T_{0}} \sum_{j=0}^{h-1}\left[\mathbf{M}_{l j}+\omega_{s, j}^{2} \delta_{j l}\right] \hat{r}_{j} .
$$

The eigenvalue $\lambda$ is then given by

$$
\lambda=\left(\Omega^{2}-i \frac{2 \Omega}{\mathcal{T}_{0}}\right) .
$$

The resonant frequency of the corresponding eigenmode can then be found as

$$
\operatorname{Re}(\Omega)=\sqrt{\frac{\operatorname{Re}(\lambda)-1 / \mathcal{T}_{0}^{2}+\sqrt{\left(\operatorname{Re}(\lambda)-1 / \mathcal{T}_{0}^{2}\right)^{2}+\operatorname{Im}(\lambda)^{2}}}{2}}
$$

and the total growth rate is

$$
\operatorname{Im}(\Omega)-\frac{1}{\mathcal{T}_{0}}=\frac{\operatorname{Im}(\lambda)}{2 \operatorname{Re}(\Omega)} .
$$

It can be noted from Eq. (16) and Eq. (17) that inclusion of the radiation damping makes little difference beyond reduction of the evaluated mode growth rate, provided that one period of oscillation is much shorter than the radiation damping time and consequently $\operatorname{Re}(\lambda) \gg 1 / \mathcal{T}_{0}^{2}$. This is neglecting all diffusion effects as previously stated.

In summary, three aspects are included here that make the theory more general than in the references mentioned in the Introduction [4,6-8]. The first is the use of the individual complex form factor for each bunch which allows the accommodation of any bunch profile with a single synchronous phase and accounts for variation in the bunch lengths and longitudinal profiles along the train. The second is that the assumption $\Omega \approx\left\langle\omega_{s, l}\right\rangle$ is not made and this allows for large shifts in the coherent frequency of coupled-bunch modes from the incoherent synchrotron frequency averaged over all bunches. Finally, at no point is the assumption made that the bunches are equally separated in time. This is usually required for a conversion to the frequency domain. Here, however, no conversion to the frequency domain is attempted.

Once the eigenvalue-mode pairs have been determined, it may be desirable to interpret them in the frequency domain in order to assign numbers to the unstable coupled-bunch modes and compare with experimental observations. This can be done with a discrete Fourier transform of the eigenvector $\hat{r}_{l}$, perhaps weighted by the bunch charges, but it should be noted that this does not give an accurate frequency-domain representation of the eigenmode in cases where the bunches are not equally spaced in time. In addition, unlike with a uniform fill, it will typically not be possible to fully describe the eigenmode by associating it with a single revolution harmonic. Nevertheless, an approximate frequency spectrum can be obtained, which may be compared to the results of applying similar operations to experimental data.

As will be shown later, the assumption of harmonic motion holds well, even with considerable Landau-cavity fields. However, unlike the method outlined in Sec. II A, validity is lost when the Landau-cavity fields are very large so that the rf potential becomes flat at the equilibrium time offset. The assumption of harmonic motion then begins to break down and it becomes difficult to define a single incoherent synchrotron frequency for the bunch. When the bunches are overstretched and there are two equilibrium time offsets, the method certainly becomes invalid.

\section{STATIC TRANSIENT}

The method to determine static transients has been extensively benchmarked as reported in [5] and its predictions 


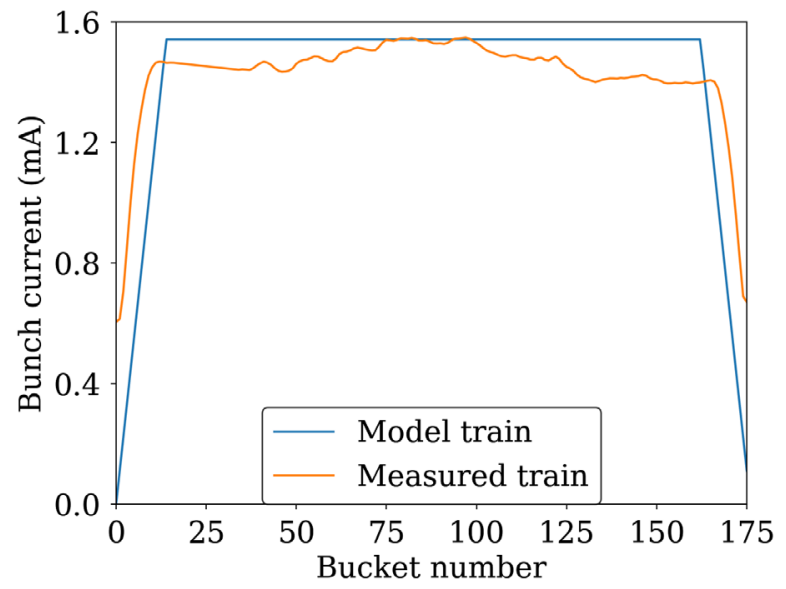

FIG. 1. Bunch-train current profiles used in the reconstruction of the beam transient.

have been compared to measurements of modest beam transients at the MAX IV $3 \mathrm{GeV}$ ring. Here, its predictions are compared to more recent measurements of a much larger beam transient also at the MAX IV $3 \mathrm{GeV}$ ring where a nonuniform fill pattern is used to reliably prevent HOMdriven instabilities. The machine parameters are listed in Table I and are the same as those currently used for actual delivery of light to users (except that the energy loss per turn is without insertion devices, which were fully open during the experiment so that their impact was negligible). The exact fill pattern is initially determined by the method used for injection. Each pulse of the full-energy linac injector accelerates a train of bunches whose length corresponds to roughly nine rf buckets in the ring. After each train is injected, the delay of the linac relative to the ring revolution trigger is stepped so as to distribute the injections among the different ring rf buckets. This process continues until the delay exceeds the requested number of buckets, at which point, it wraps around and steps the remainder at the beginning of the train so that the process does not always start from zero. The step in delay is deliberately chosen to not be a factor of the number of buckets requested to avoid seeing a periodicity in the bunch charges in the ring that mirrors the charge profile of the linac train. Tapers in bunch charge then arise at the ends of the bunch train in the ring because these buckets are injected into less often than the others. In addition, the different bunches in the train have different bunch lengths and therefore, different Touschek lifetimes, which means that after several top-up injections, the longer bunches have more charge in them than the shorter bunches. The fill pattern, as measured by integrating the sum signal of a BPM, is shown in Fig. 1 where a simple model bunch train which also features tapering of the bunch charge at each end is also shown. The model train is introduced here because it is later used to test the theory outlined in Sec. II B. It is used instead of the measured train for this purpose because it is free from noise which makes the predictions of the theory easier to interpret. It should be noted that the temporal resolution of the BPM measurement is not

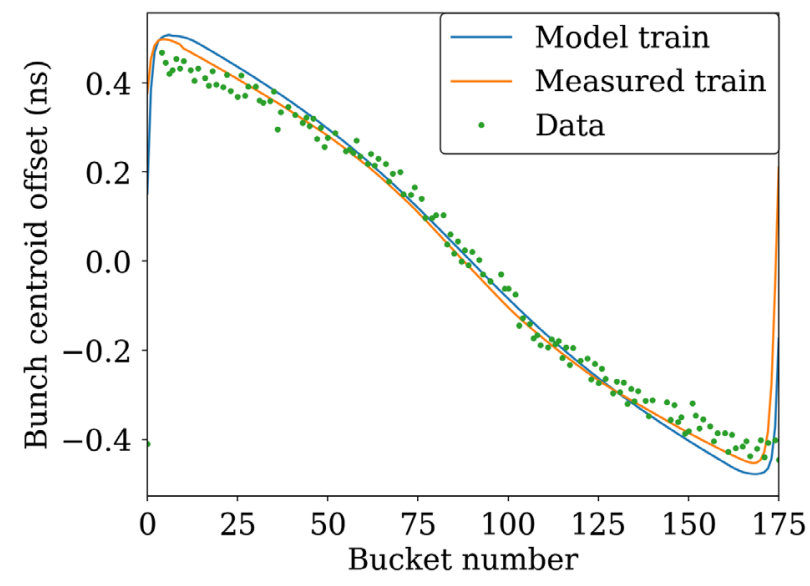

FIG. 2. Comparison of the predicted beam transient for different train current profiles with measured bunch time offsets.

sufficient to accurately resolve the charge of each individual bunch and so some smoothing of the train current profile is expected. For example, it is known from the optical sampling oscilloscope measurements introduced below, that one bucket contains practically zero charge and this does not show up in the BPM-based charge measurement.

Figure 2 shows the measured beam transient in comparison to the prediction from the theory outlined in Sec. II A. An optical sampling oscilloscope from Hamamatsu was used to take measurements of longitudinal bunch profiles, from which the measured data are extracted. The detector head of this device has a streak tube for the sampling followed by a photomultiplier tube. This is not a single-shot measurement and multiple turns are required to acquire each bunch profile. To acquire the whole train took close to two hours with regular pauses for top-up injections. Furthermore, not all profile measurements were successful, most likely due to a feedback used to compensate for thermal drifts in the experimental setup, which moved a mirror motor during the sweep of the oscilloscope. These points were identified from the difference in the extracted bunch length to those of the neighboring buckets and in total, 136 out of 176 data points were kept. All the results in this section are from the same series of bunch-profile measurements.

In the calculation, the beam-loading of the main cavities was neglected. This is justified because the time derivative of the beam-loading field in the main cavities is much smaller due to the lower frequency and smaller tuning angle. Predictions have been made for both the model bunch train and the measured bunch charges assuming a Landau cavity detuning of $+60 \mathrm{kHz}$, which was found to well reproduce the measured data. This is slightly smaller than the detuning that would be used to obtain flat potential with a uniform fill. The predicted transient based on the measured bunch charges is less steep at the ends of the train than the one based on the model and it is also slightly closer to the measured data. This is a consequence of the higher 


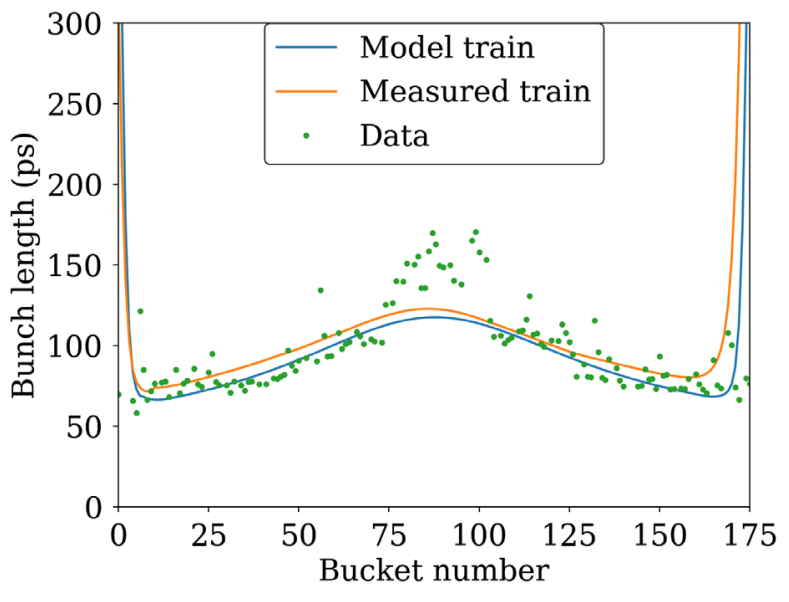

FIG. 3. Comparison of the predicted bunch lengths for different train current profiles with measured values.

charges stored in the longer bunches in the middle of the train. Both predictions overestimate the measured transient and this is likely due to inaccuracies in the train current profile, since the difference between the two predictions is of similar magnitude to the discrepancy. Alternatively, it could be because of the profiles of the bunches in the taper, which tend to be overstretched to the extent that they consist of two distinct bunches. These profiles are not so well reproduced by the theory, as discussed later, and this could also be a cause of the aforementioned discrepancy. It is also not excluded that multiple, physical solutions exist to the problem. Nevertheless, the agreement between measurement and theory is good.

Figure 3 shows the bunch lengths along with predictions made for the same two bunch trains as in Fig. 2 using the theory outlined in Sec. II A. Results corresponding to the same 136 data points that appear in Fig. 2 are included and they show a smooth variation in the bunch length along the train, which is well reproduced by the theory. However, the measured data also include variations with a shorter period. These are physical and caused by the transient beam loading of one or more higher-order modes in the cavities as discussed in [5]. Finally, the underestimation of the bunch lengths in the middle of the train is most likely due to residual coupled-bunch motion, which has been detected but which is not large enough to have a measurable impact on the energy spread.

As demonstrated in [5], the theory is not only able to reproduce beam transients and bunch lengths but also the precise bunch profiles. Several examples of this are shown in Fig. 4, where the measured bunch train has been used. For certain bunches, the two profiles appear to be displaced from one another in time. This is a result of the theory overestimating the beam transient as mentioned previously. Overall, however, the agreement between the theory and the measurement is very good. In the tapers of the train where the bunches are overstretched (bunch 171, for example), the theory correctly predicts the existence of two distinct bunches but does not correctly predict their separation or relative sizes. Still, the profile of the larger bunch lines up with the theory very well. One assumption made in the theory is that the beam loading field does not change significantly over the length of one single bunch. This assumption may explain the difference in the case of an overstretched bunch such as this one because small differences in the beam loading can lead to large differences in the bunch profile.

\section{BENCHMARKING}

Before moving on to evaluate the growth rates of coupled bunch modes in the presence of the transient evaluated in Sec. III, the method of determining growth rates was benchmarked against the macroparticle tracking code, MBTRACK, for the simple case of a uniform fill pattern, a single HOM and no Landau cavities. MBTRACK has previously been benchmarked against theory [20]. The machine parameters in Table I were used and in MBTRACK, one macroparticle per bunch was selected so that no discrepancy arose due to the dynamics within each bunch, which are not treated by the theory. The HOM used in the simulation is close to the revolution harmonic at $1.09413 \mathrm{GHz}$ so that it drives coupled-bunch mode

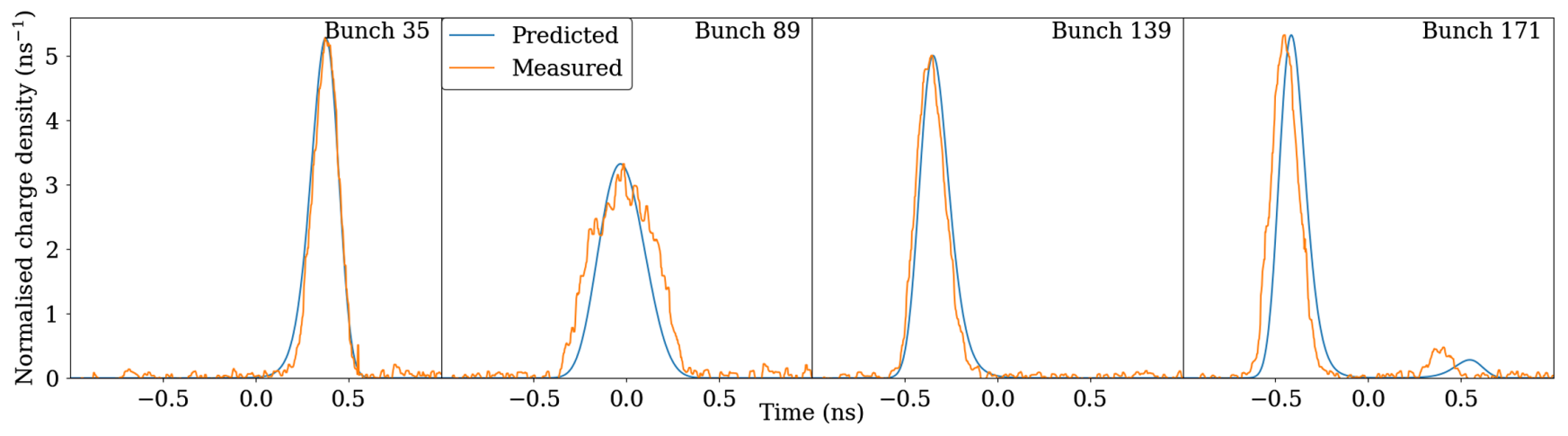

FIG. 4. Four different bunch profiles as measured using the synchrotron light in comparison with profiles predicted by the theory taking the measured bunch train as an input. 


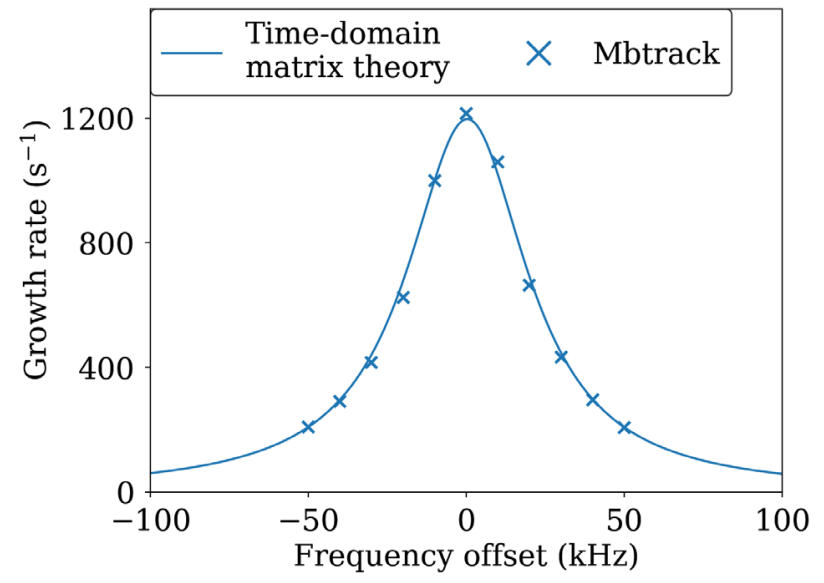

FIG. 5. Growth rates predicted for a uniform fill with point bunches compared to results obtained using MBTRACK. The horizontal axis is the offset in frequency of the HOM from the closest revolution harmonic at $1.09413 \mathrm{GHz}$.

number 167. It is based on a HOM that has been measured in one of the cavities in the MAX IV $3 \mathrm{GeV}$ ring and that also shows up in electromagnetic simulations. The quality factor of 24000 was obtained from transmission measurements on the cavity using a network analyzer. According to electromagnetic simulations, the HOM in question should then have shunt impedance of $325 \mathrm{k} \Omega$ but for the benchmarking, a value of $160 \mathrm{k} \Omega$ was used so that the growth rate on resonance was small enough to be easily extracted from the results of the macroparticle simulations. This was done by demodulating the time-domain data by sampling the positive and negative peak time offsets of each bunch every synchrotron period, averaging the absolute values over all bunches, taking the natural logarithm and then performing a linear fit. Radiation damping was neglected for the purposes of this benchmarking. Figure 5 shows that the time-domain matrix method predicts very similar growth rates to those obtained using MBTRACK for the same HOM parameters.

Next, the model nonuniform fill from Sec. III was simulated in the presence of the same HOM, still with only one macroparticle per bunch in the tracking and neglecting the form factor in the theory. The Landau cavities were also reintroduced with a detuning of $+64 \mathrm{kHz}$. The synchrotron frequency of each bunch $\omega_{s, l}$ was calculated from the derivative of the local total $\mathrm{rf}$ voltage, as described in [5]. The spread in synchrotron tune has a large effect and so here, the calculation of the incoherent synchrotron tune for each bunch is important. The results of this calculation are shown in Fig. 6.

In this scenario, the bunch motion is highly nonlinear and the growth of the coupled-bunch modes is not exponential, which means that it is not possible to determine the growth rates from the MBTRACK results using the method described above. Instead, the theoretical calculation was iterated to obtain numerically a threshold value for the

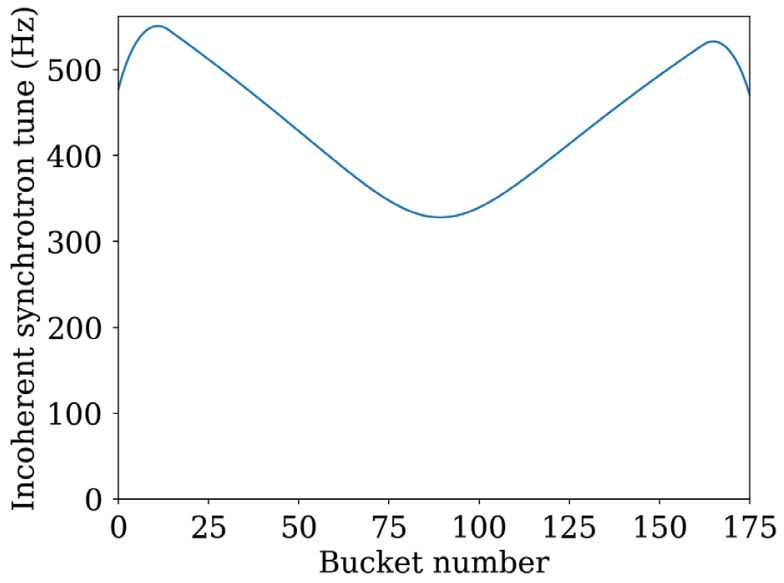

FIG. 6. The synchrotron frequency calculated for each bunch from the derivative of the total local rf voltage.

shunt impedance above which, the least stable coupledbunch mode has a growth rate that is larger than the radiation damping rate. Radiation damping was also included in the macroparticle simulations, although quantum excitation could not be included because there is only one macroparticle per bunch and a coherent excitation would be unphysical. The results are shown in Fig. 7. It can be seen that, once again, the theory agrees well with the macroparticle simulations. However, the agreement is not perfect, particularly when the HOM frequency is lower than the nearest revolution harmonic. Two assumptions made in the theory might explain this difference. The first is that the motion of the bunches is perfectly harmonic and the second is that the HOM has no effect on the static transient or the incoherent synchrotron frequency when in reality and in the MBTRACK simulations, it does have a static effect, albeit a small one. It is actually possible to include this effect in the theory by including the HOM in the calculation of the static

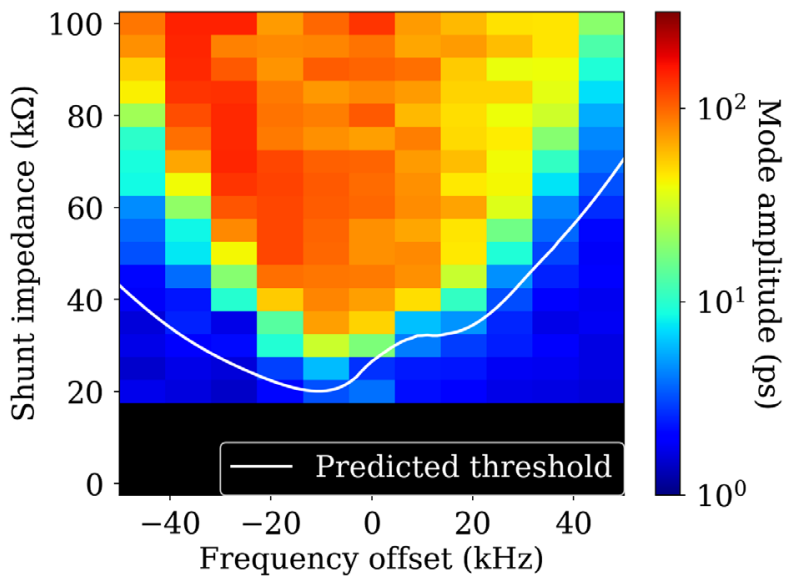

FIG. 7. Bunch motion observed in macroparticle tracking compared to the threshold shunt impedances predicted by theory for point bunches. The black pixels are points for which no simulation was run. 


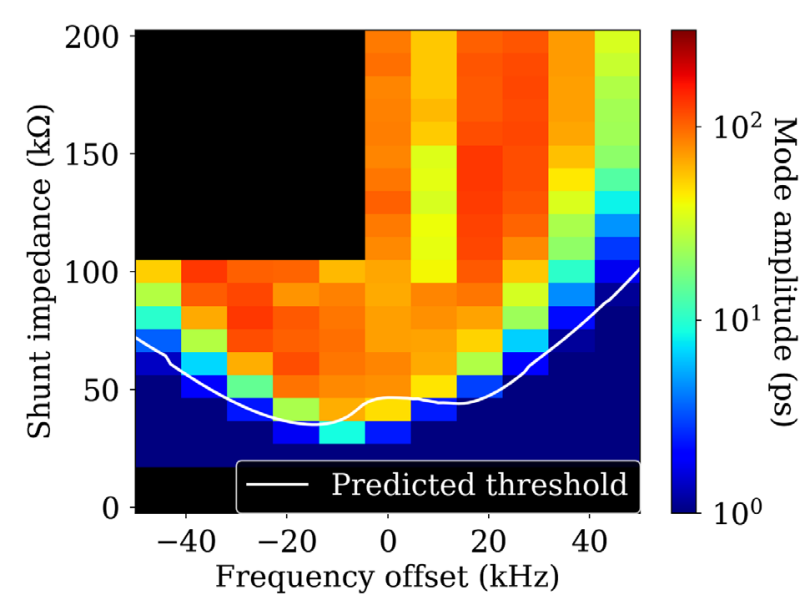

FIG. 8. Bunch motion observed in macroparticle tracking compared to the threshold shunt impedances predicted by theory including the effect of the bunch forms and lengths. The black pixels are points for which no simulation was run.

transient. However, this would increase the calculation time significantly because the transient would have to be reevaluated for every HOM detuning and shunt impedance. If the HOM is neglected in the calculation of the static transient, this calculation only needs to be performed once. Evaluating the coupled-bunch modes, which is far less demanding than the static-transient calculation in terms of computing time, can then be done separately for each HOM detuning.

It can be seen in the results of both the macroparticle simulations and the theory that when the HOM is tuned to lower frequencies than the nearest revolution harmonic, the threshold shunt impedances are slightly lower than at the same positive frequency offsets. This arises from the interplay between the real and imaginary part of the coherent frequency shift in Eq. (16) and Eq. (17). If Eq. (13) is used instead, no such asymmetry is obtained, which highlights the importance of not making this common approximation in this case. A similar asymmetry was observed in [15].

Figure 8 shows the results of the same calculation where the finite bunch size has been included in both the simulation, through increasing the number of macroparticles per bunch to 10000 , and in the theory by including the complex form factors of each bunch. Here, quantum excitation is additionally included in the macroparticle simulation to stop the energy spread in the bunch going below the nominal value. One difference with the results shown in Fig. 7 is that the threshold shunt impedances are slightly higher across the full frequency range because the bunch form factors are less than unity. The inclusion of a finite bunch length has improved the level of agreement compared to the simpler case where the bunch form factors were neglected. The level of agreement justifies the assumptions and approximations made, including in the calculation of the incoherent synchrotron frequency for each bunch.

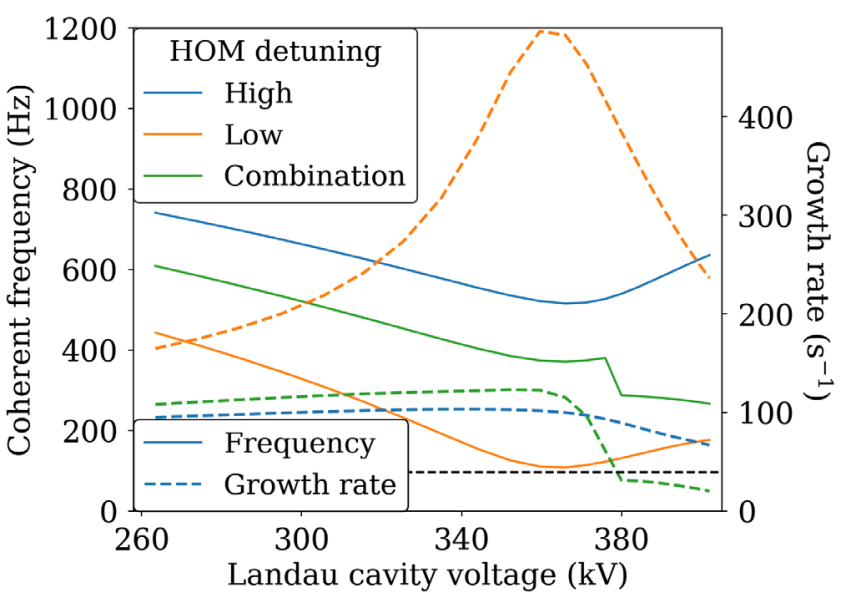

FIG. 9. Growth rates and coherent frequencies of coupledbunch modes driven by two similar HOMs either detuned similarly or in opposite directions from the nearest revolution harmonic. The black dashed line shows the radiation damping rate of $40 \mathrm{~s}^{-1}$.

\section{EFFECT OF LANDAU-CAVITY FIELDS}

To illustrate the utility of the developed method, it was applied to evaluate the effect of the HOM used in Sec. IV, with the more accurate shunt impedance of $325 \mathrm{k} \Omega$, on the delivery conditions at the MAX IV $3 \mathrm{GeV}$ ring. The effect of this HOM was estimated using the theory outlined in Sec. II B for different values for the detuning of the Landau cavities. It was assumed that the same HOM was present in two of the main cavities and could be detuned independently, as is achieved in reality by using different operating temperatures for each cavity. Again, the beam loading of the fundamental mode in the main cavities is neglected, as is the effect of the HOM on the static transient. Figure 9 shows the predicted growth rate and coherent frequency of the fastest-growing coupled-bunch mode for three different scenarios. The blue and orange curves correspond to cases in which both HOMs are detuned by $175 \mathrm{kHz}$ in the same direction, higher or lower than the neighboring revolution harmonic (respectively referred to as "high" and "low" in the legend). The green curves are for the case in which the two HOMs are detuned by $175 \mathrm{kHz}$ but in opposite directions (referred to as "combination" in the legend). As could be expected from the results in Sec. IV, the growth rates are larger when both HOMs are detuned to lower frequencies than when both are detuned to higher frequencies.

In all three scenarios, above a certain Landau-cavity voltage, the coherent frequencies start to increase and the growth rates start to decrease. The interplay between the coherent frequency and the growth rate of the coupledbunch modes is not surprising because the two are linked according to Eq. (16) and Eq. (17). The decrease in the growth rate is particularly sharp in the case of the two HOMs being detuned in opposite directions. In this 


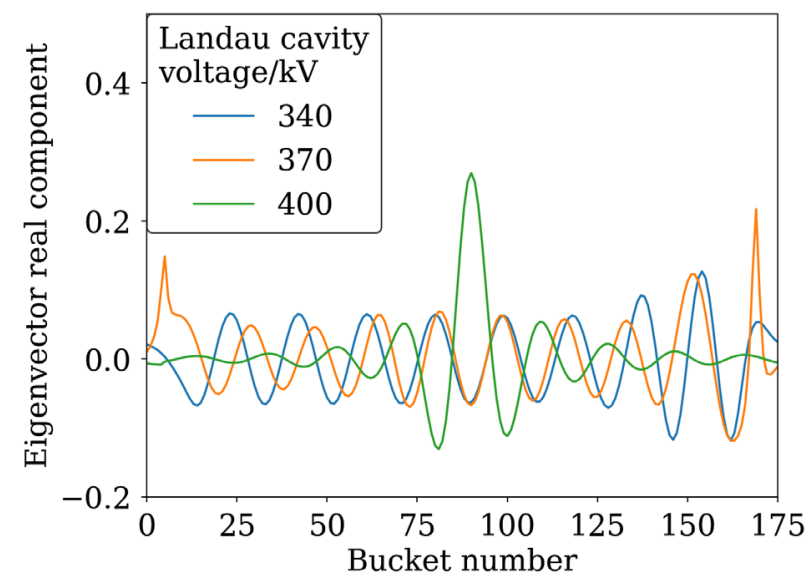

FIG. 10. The real component of normalized eigenvectors that correspond to the least stable coupled-bunch mode at three of the different Landau-cavity voltages that appear in Fig. 9 for the case of two HOMs tuned differently.

scenario, the growth rate goes below the radiation damping time and reaches the lowest values shown in the figure. At higher Landau-cavity voltages, there is a discontinuity in the curve of the coherent frequency of the least-stable coupledbunch mode. The reason for this discontinuity is that in addition to changing the eigenfrequencies, the change in the Landau-cavity voltage also changes the eigenmodes themselves, as shown in Fig. 10. When the Landau-cavity voltage is lower than $377 \mathrm{kV}$, the point of the discontinuity on the horizontal axis, the least-stable eigenmode maintains a somewhat constant form where all bunches oscillate with roughly the same amplitude. At higher Landau-cavity voltages, on the other hand, the form is markedly different: the coupled-bunch motion is mostly confined to the bunches in the middle of the train. At Landau-cavity voltages below $370 \mathrm{kV}$, detuning both HOMs to higher frequencies delivers the lowest growth rates.

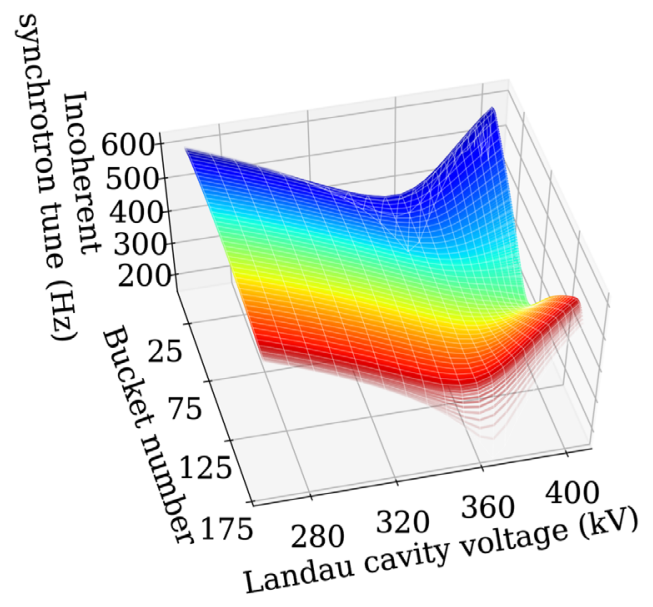

The behavior shown in Fig. 9 can be understood by looking at the incoherent frequency of the bunches in the train, which are shown in Fig. 11. The incoherent synchrotron frequencies are obtained from the results of the statictransient calculation and so, since the HOMs were neglected in this calculation, the same frequencies (as well as bunch profiles and lengths) were used in the second step for all three scenarios. To begin with, for increasing Landau-cavity fields, the incoherent frequency is decreasing for all bunches, same as for the coherent frequency of the least stable coupled-bunch modes in Fig. 9. Then, at just above $360 \mathrm{kV}$ of Landau-cavity voltage, the incoherent synchrotron tunes of the bunches close to the ends of the bunch train start to increase while the tunes of the middle bunches continue to decrease. This is accompanied by a significant increase in the spread in synchrotron frequency to almost $100 \mathrm{~Hz}$ RMS.

As pointed out in [16], for Landau damping to occur, the "frequency of the [coupled-bunch] mode of interest should overlap with the system natural frequency spread." We can see by comparing Fig. 9 with Fig 11 that this is only true in the case of the two HOMs being tuned to opposite sides of the nearest revolution harmonic. This is because, in this case, the reactive impedances of the two HOMs cancel and so the resulting frequency shift is close to zero. Landau damping is therefore only realized in this case. This is an important conclusion that should be kept in mind when dealing with HOMs in rf systems with multiple cavities.

In order to justify neglecting intrabunch Landau damping, the tune spread within each bunch was calculated using the theory in the appendix of [9] for the highest Landau-cavity voltage shown in Fig. 9 and is compared with the interbunch tune spread in Fig. 12. For all bunches, the intrabunch tune spread is below $16 \mathrm{~Hz}$ and the average value (weighted by bunch charge), is $10 \mathrm{~Hz}$. As can be seen on the figure, this is almost a

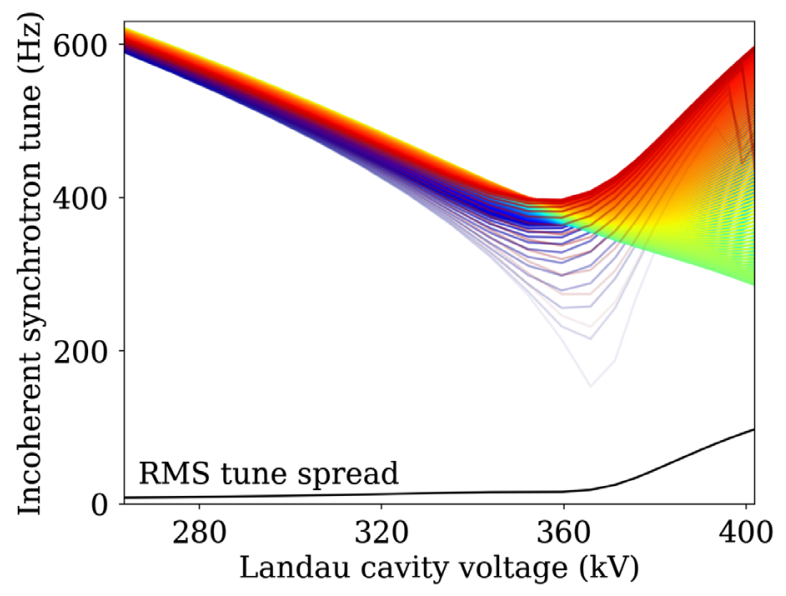

FIG. 11. Incoherent synchrotron tune spread within the bunch train as a function of Landau cavity voltage: 3D plot on the left and projection on the right. 


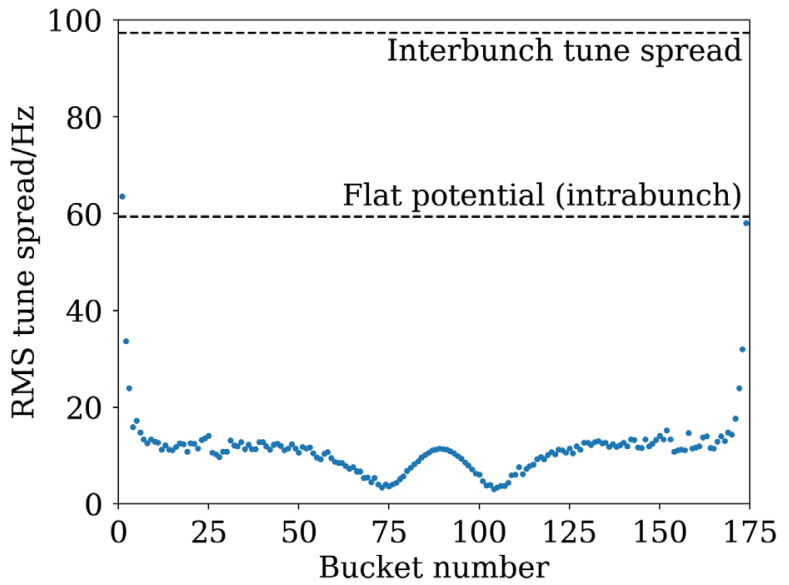

FIG. 12. Intrabunch RMS synchrotron tune spread within each bunch with $402 \mathrm{kV}$ Landau-cavity voltage.

factor of ten smaller than the tune spread between bunches for the same nonuniform fill and a factor of six smaller than the intrabunch tune spread when flatpotential conditions are satisfied. Comparing the tune spreads in this way is not an accurate comparison of the amount of damping present (to what extent this can be done is discussed in [15]) but it does support the assumption that, for this particular nonuniform fill, the damping due to the frequency spread between bunches is dominant. This assumption is also supported by the good agreement shown in Fig. 8 between macroparticle tracking, a method that includes both intrabunch and interbunch tune spreads and theoretical predictions that neglect the intrabunch component. It is likely that this assumption applies more generally to nonuniform fills where the bunch-lengthening is significantly reduced compared to flat-potential conditions, although each case should be evaluated individually.

As well as being a turning point for the incoherent synchrotron frequency, for Landau-cavity voltages above $370 \mathrm{kV}$, the bunches in the middle of the train are longer than those at the edge, as shown in Fig. 13. A similar phenomenon was observed in [5], although in that case it was the length of the bunch train that was being varied.

Another advantage of the method used here to evaluate the growth rates is the ease with which certain effects can be separated so that the impact of each can be gauged, even if it is not possible in reality to maintain one of the effects without eliminating the other. The results of such an exercise for the third case in Fig. 9 are shown in Fig. 14. It can be seen that the aspect that has the largest impact is the tune spread between the bunches. When this is removed, the steep drop in growth rate when the Landau cavity field is above $360 \mathrm{kV}$ is not seen and instead, the decrease is more gentle, similar to in the other two cases in Fig. 9. It is only when the tune shift due to the increase

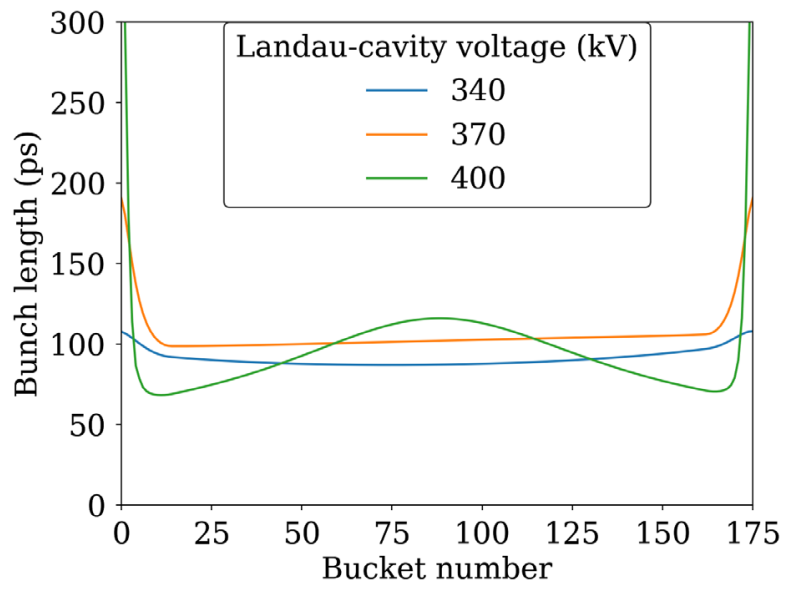

FIG. 13. Bunch length for different bucket numbers for three different Landau-cavity voltages that appear in Fig. 9.

in Landau cavity field is also removed that the effect of the bunch lengthening shows up. In this case, the growth-rate curve mimics the change in the average form factor of the bunches in the train and is at a minimum around $370 \mathrm{kV}$ Landau cavity voltage where all of the bunches have roughly the same length. Another case that was tried was removing the phase transient by giving all the bunches the same time offset. This is not shown in the figure as it was found to make very little difference for the following reason. In a coupled-bunch mode in a uniform fill, there is a fixed phase advance between the oscillations of consecutive bunches. In a nonuniform fill, on the other hand, the

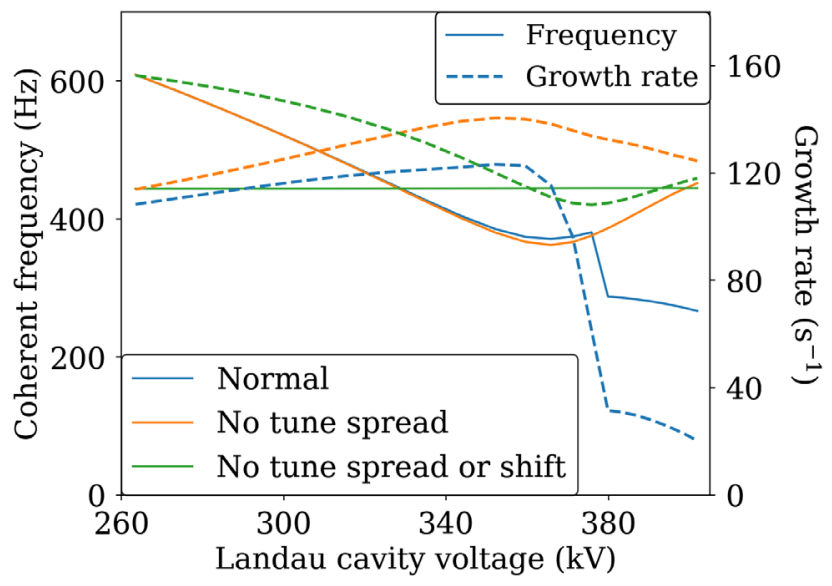

FIG. 14. Growth rates and coherent frequencies of coupledbunch modes driven by two HOMs detuned in opposite directions from the nearest revolution harmonic. The impact of two effects are investigated. First, the tune spread is removed by setting the tune of each bunch to the average value $\left\langle\omega_{s}, l\right\rangle$. Second, the tune shift due to the increased fields in the Landau cavities is removed by setting the tune of each bunch to the average value for all bunches and all Landau cavity fields in the figure. The cases are otherwise identical. 


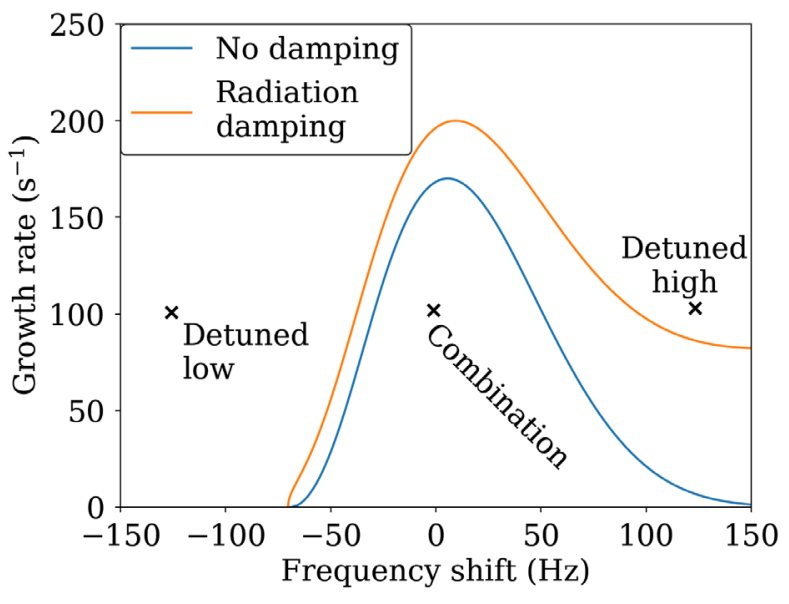

FIG. 15. Growth rates against frequency shift of coupled-bunch modes driven by two similar HOMs either detuned similarly or in opposite directions from the nearest revolution harmonic for bunches in a flattened rf potential. The points correspond to results for the three different cases while the curves are stability contours: anything below the curve is stable due to Landau damping.

phase advance varies because the temporal separation from one bunch to the next is not fixed. This varying phase advance allows the HOM to remain approximately resonant with the beam, even though the transient is large compared to the bandwidth of the HOM.

For comparison, calculations for the same HOMs have been performed for the case of a uniform fill with the flat-potential condition satisfied, which can be achieved with a Landau-cavity voltage of $397 \mathrm{kV}$. The rf potential around each bunch is then quartic [1] and the RMS synchrotron-tune spread within each bunch is $60 \mathrm{~Hz}$, as shown in Fig. 12 and there is no tune spread between the bunches. The theory for this case as presented in [15] has been applied and the results are shown in Fig. 15. The theory assumes that the tune shift is small and for the cases where both HOMs are tuned to the same side of the resonance, this is clearly not the case. This approximation is also the reason that for all three cases, the same growth rate is predicted in the absence of Landau damping. Nevertheless, similar to the nonuniform-fill case, when the HOMs are tuned to the same side of resonance, their reactive impedance means that the coherent tune of the least-stable coupled-bunch mode is outside of the natural tune spread within the bunch and so the beam is more likely to be unstable. When the two HOMs are tuned differently, their reactive impedances cancel so the coherent tune shift is small and the Landau damping is strong. In this case, the results predict that the Landau damping provided by a quartic potential is also enough to prevent a coupled-bunch mode from being unstable.

A uniform fill and the flat-potential condition is the antithesis of the case of a nonuniform fill with transient beam loading in that it is the intrabunch tune spread that dominates where as in the latter, it is the interbunch tune spread that dominates. However, it is not excluded that a situation could arise where both effects are equally important and in this case, neither the theory presented in Sec. II B nor the theory used to produce Fig. 15 would be sufficient to solve the problem completely. The appendix of [15] does discuss how one would go about doing this, particularly for the case of two or more bunch trains, but it does not go so far as to evaluate the dispersion relation for a realistic scenario and for the specific cases that are explored in depth in [15], namely harmonic motion, weakly nonlinear potential and flat potential, the evaluation involves some loss of generality and the assumption of a small coherent tune shift.

\section{CONCLUSION}

A method has been developed to accurately evaluate the stability of coupled-bunch modes in a synchrotron storage ring in the presence of an arbitrary fill pattern. The method builds upon and extends previous work and evaluates not only the static but also the dynamics effects of nonuniform fills accounting for the uneven spacing of the different bunches and their different charge profiles. The transient beam loading, which is particularly pronounced when Landau cavities are in use, leads to a spread in the synchrotron tune between bunches and this can damp coupled-bunch instabilities. The first step in the new method is to evaluate a selfconsistent solution for the effects of the transient beam loading in terms of the phase transients and the variation in the lengths and profiles of the different bunches. This is done through the use of a complex form factor and produces results that agree well with experimental observations. From these results, the growth rates and coherent frequencies of the different coupled-bunch modes can then be calculated directly. The ease of the transition from the first step to the second can be seen in the similarities of the matrices in Eq. (1) and Eq. (11) which mean that in practice, computer code used to compute the result of the first step can be reused in the second. The profile of each individual bunch is also accounted for in the second step through the use of individual complex form factors.

As an alternative, input to this second calculation could be obtained from macroparticle tracking or experiment, either of which could be used to determine the equilibrium time offsets of the bunches and their longitudinal profiles. However, both of these sources are affected by noise and the discretization may not be optimal for calculation of the bunch form factors so additional post-processing would be inevitable. Furthermore, as mentioned in [5], all steps in this method can be carried out on a laptop or personal computer in contrast to macroparticle simulations, which 
typically require computing clusters when dealing with multiple bunches. The total computation time for the new method is dominated by the first step so the comparison made in [5] is also valid here (5.5 minutes on a laptop compared to 28 minutes on a node with 20 cores for the macroparticle tracking). Within this, the numerical integration to determine the longitudinal bunch profiles and form factors is the most time consuming so the computation time increases almost linearly with the number of $\mathrm{rf}$ buckets. Once the first step is complete, the evaluation of the growth rates and coherent frequencies takes less than a third of a second for MAX IV. This would increase with the square of the number of buckets but a machine would have to have over $2000 \mathrm{rf}$ buckets for the computation to take more than one minute. There is, therefore, a lot to be gained in terms of computing resources from using this method instead of macroparticle tracking, particularly when evaluating the effects of HOMs in large storage rings.

The extended method for evaluating dynamic effects has been thoroughly benchmarked against the results of macroparticle tracking simulations. For cases with nonuniform fill patterns, the threshold shunt impedances of a HOM driving coupled-bunch modes were calculated for the comparison. This gives confidence, not only in the accuracy of the results, but also that the new method can be used alongside macroparticle tracking, making the most of the advantages that the former has with regards to computing resources and that the latter has with regards to comprehensive inclusion of multiple phenomena.

Finally, the method has been used to investigate the effects of selective HOM tuning on coupled-bunch mode stability. It is predicted that, in the case of the same HOM existing in two cavities, it is beneficial to tune the HOM differently in each when the Landau cavity field is high. This is because the reactive impedances cancel and so the coherent frequency is within the spread of the natural frequencies of the system, which is a necessary condition for Landau damping [16]. This raises the more general point that in any system that relies on Landau damping for stability, the reactive impedance should be minimized because the system will lose stability if there is a large shift in the coherent tune. At low Landau-cavity fields, tuning both HOMs to higher frequency is beneficial. To get a more accurate prediction for a given ring, the exact nature of the HOMs in all cavities must be known. Work is currently ongoing at MAX IV to obtain such a model for the cavities in the $3 \mathrm{GeV}$ ring [21].

\section{ACKNOWLEDGMENTS}

The authors would like to thank Mikael Eriksson for stimulating discussions and much besides. The MBTRACK simulations were carried out on the MAX IV computing cluster, for which we thank the members of the controls and IT division.
[1] A. Hofmann and S. Myers, Beam dynamics in a double RF system, Report No. CERN-ISR-TH-RF-80-26, 1980, http://cds.cern.ch/record/879237.

[2] S. C. Leemann, Interplay of touschek scattering, intrabeam scattering, and rf cavities in ultralow-emittance storage rings, Phys. Rev. Accel. Beams 17, 050705 (2014).

[3] G. Penco and M. Svandrik, Experimental studies on transient beam loading effects in the presence of a superconducting third harmonic cavity, Phys. Rev. Accel. Beams 9, 044401 (2006).

[4] O. Naumann and J. Jacob, Fractional filling induced Landau damping of longitudinal instabilities at the ESRF, in Proceedings of the 1987 Particle Accelerator Conference (IEEE, Washington, DC, 1987), pp. 1551-1553.

[5] T. Olsson, F. J. Cullinan, and A. Andersson, Self-consistent calculation of transient beam loading in electron storage rings with passive harmonic cavities, Phys. Rev. Accel. Beams 21, 120701 (2018).

[6] K. A. Thompson and R. D. Ruth, Transverse and longitudinal coupled bunch instabilities in trains of closely spaced bunches, in Proceedings of the 1989 Particle Accelerator Conference, Chicago, IL, Vol. 2 (IEEE, New York, 1989), pp. 792-794.

[7] G. Bassi, A. Blednykh, and V. Smaluk, Self-consistent simulations and analysis of the coupled-bunch instability for arbitrary multibunch configurations, Phys. Rev. Accel. Beams 19, 024401 (2016).

[8] K. A. Wang, P. J. Chou, and A. W. Chao, Study of nonuniform fills to cure the coupled-bunch instability in srrc, in Proceedings of the 19th Particle Accelerator Conference, Chicago, IL, 2001 (IEEE, Piscataway, NJ, 2001), pp. 1981-1983.

[9] P. F. Tavares, Å. Andersson, A. Hansson, and J. Breunlin, Equilibrium bunch density distribution with passive harmonic cavities in a storage ring, Phys. Rev. Accel. Beams 17, 064401 (2014).

[10] F. J. Cullinan, R. Nagaoka, G. Skripka, and P. F. Tavares, Transverse coupled-bunch instability thresholds in the presence of a harmonic-cavity-flattened RF potential, Phys. Rev. Accel. Beams 19, 124401 (2016).

[11] M. Sjöstrum, Status of the MAX IV accelerators. 2018. Presented at ESLS XXVI, SOLARIS National Synchrotron Radiation Centre, Krakow, Poland on Tuesday 27th November, 2018, https://synchrotron.uj.edu.pl/documents/ 1457771/141441388/06_MAXIV_Status_report_ESLS_26 .pdf/54f5546f-0b01-450c-b33b-829b10162811.

[12] D. McGinnis (private communication).

[13] G. Skripka, R. Nagaoka, M. Klein, F. Cullinan, and P. F. Tavares, Simultaneous computation of intrabunch and interbunch collective beam motions in storage rings, Nucl. Instrum. Methods Phys. Res., Sect. A 806, 221 (2016), ISSN https://doi.org/NIMAER.

[14] S. Krinsky and J.M. Wang, Longitudinal instabilities of bunched beams subject to a nonharmonic rf potential, Part. Accel. 17, 109 (1985), https://s3.cern.ch/inspire-prodfiles-5/5f44fce 5c9b28741a1bafd4819f6cc9f.

[15] R. R. Lindberg, Theory of coupled-bunch longitudinal instabilities in a storage ring for arbitrary RF potentials, Phys. Rev. Accel. Beams 21, 124402 (2018). 
[16] M. Venturini, Passive higher-harmonic RF cavities with general settings and multibunch instabilities in electron storage rings, Phys. Rev. Accel. Beams 21, 114404 (2018).

[17] R. A. Bosch, Instabilities driven by higher-order modes in a radio frequency system with a passive higher-harmonic cavity, Phys. Rev. Accel. Beams 8, 084401 (2005).

[18] P. B. Wilson and J. E. Griffin, High energy electron linacs; application to storage ring RF systems and linear colliders, AIP Conf. No. 87, 450 (1982).
[19] D. Morin, Introduction to Classical Mechanics: With Problems and Solutions (Cambridge University Press, Cambridge, England, 2008).

[20] H. Xu, U. Locans, A. Adelmann, and L. Stingelin, Calculation of longitudinal collective instabilities with mbtrack-cuda, Nucl. Instrum. Methods Phys. Res., Sect. A 922, 345 (2019), ISSN https://doi.org/NIMAER.

[21] P. F. Tavares (to be published). 